\title{
RELACION STANDING CROP ZOOPLANCTONICO Y DENSIDAD DE LARVAS DE PECES EN BAHIA DE CONCEPCION, CHILE.
}

\author{
DAGOBERTO ARCOS R.; ALEJANDRO ARON N. Y FRANKLIN CARRASCO V. \\ Departamento de Biologia Marina y Oceanografía
}

Instituto de Biología, Universidad de Concepción, Concepción, Chile

\section{SYNOPSIS}

From monthly zooplankton samples collected during October 1976 to October 1977, the seasonal variation of zooplankton standing crop and its relation to the number of fish larvae from Bay of Concepcion has been established. The zooplankton standing crop varied between $58 \mathrm{ml} / \mathrm{m}^{2}$ in August and $430 \mathrm{ml} / \mathrm{m}^{2}$ in January. The number of fish larvae in the same sample ranged from 30 larvae/m in January to 1045 larvae $/ \mathrm{m}^{2}$ in March. An inverse relationship between the zooplankton standing crop and the number of fish larvae it was checked and this relation is discussed according to the bibliographic antecedents.

Introduccion

El zooplancton adquiere una importancia directa con las larvas de peces ya que una fracción importante de él constituye el principal alimento de éstas $y$, por otro lado, algunos zooplancteres predan activamente sobre ellas. La alta fecundidad relativa de muchos peces implica un alto grado de mortalidad que ocurre, precisamente, en los estadios larvales; de ahí que sea un problema básico de la dinámica de poblaciones de peces (May, 1974).

Se ha establecido que los principales factores que afectan la presencia de las larvas de peces en el plancton son: la predación. los factores abioticos y la inanicion. Estos factores han sido analizados por varios autores y concluyen que la predación del macro- zooplancton es la causa más importante y que afecta la densidad de las larvas en el plancton (Hjort, 1914; Marr, 1956; Hempel, 1965).

\section{Material y Metodos}

El material y las observaciones usadas en el presente trabajo provienen de muestreos mensuales efectuados en Bahía de Concepción por el B/C Lund (Fig. 1), utilizando para ello una red de zooplancton de $50 \mathrm{~cm}$ de boca y de $0,365 \mathrm{~mm}$ de trama. En el laboratorio se procedió a separar las muestras y a medir el volumen desplazado (standing crop zooplanctónico) según el método de Ali Khan (1974).

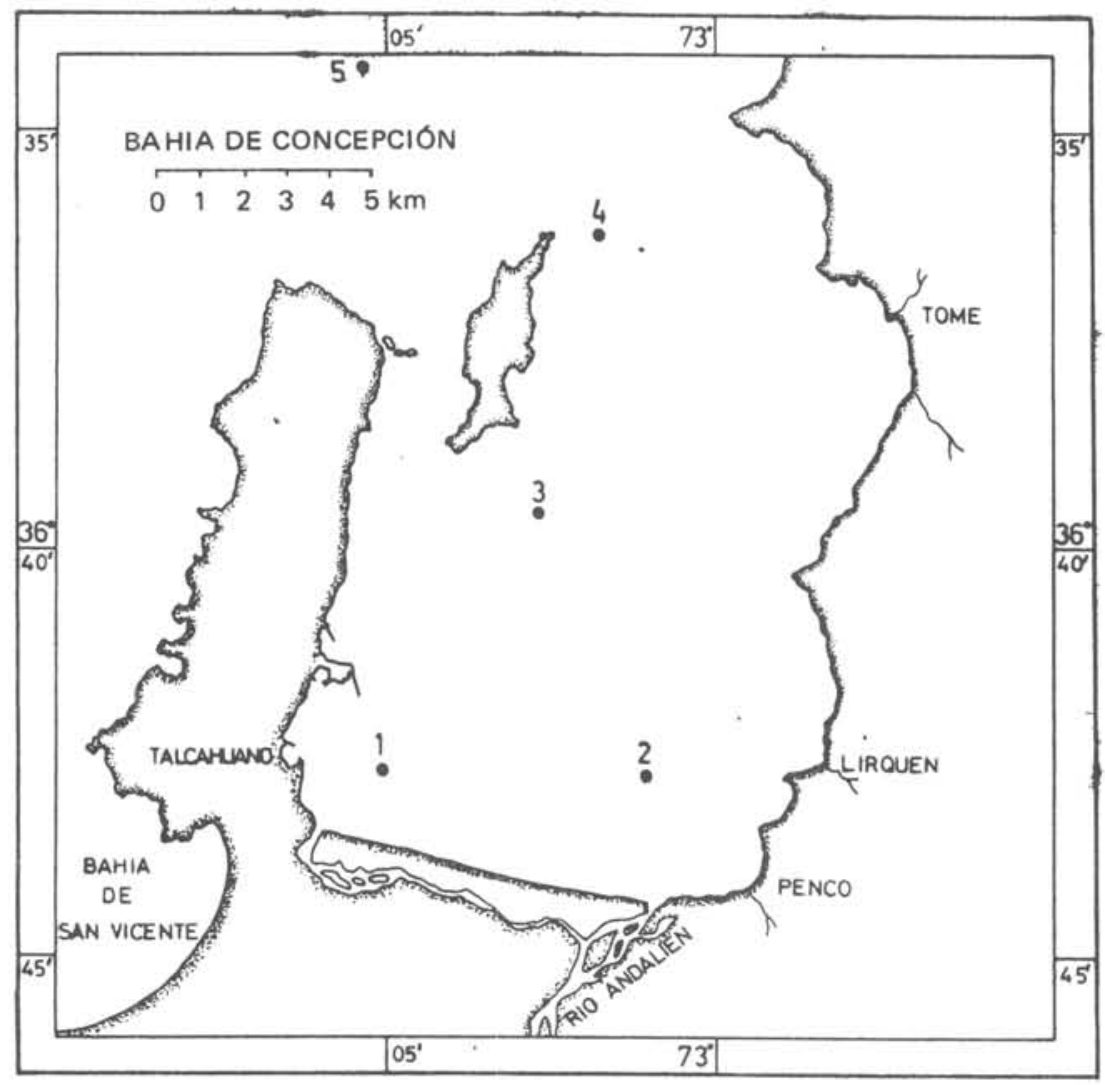

Fig. 1-Bahía de Concepción y ubicación de los lugares de muestreo.

\section{Resultados}

El standing crop zooplanctónico (SCZ) asi medido y expre sado en mililitros de zooplancton por metro cuadrado de superficie del océano, presentó variaciones entre 58 y $430 \mathrm{ml} / \mathrm{m}^{2}$ en $l o s$ meses de agosto y enero, como promedio de las estaciones utilizadas; $2 \mathrm{ml} / \mathrm{m}^{2}$ es el valor mínimo observado en el mes de octubre y de $1143 \mathrm{ml} / \mathrm{m}^{2}$ el valor máximo para el mes de enero. Por otro lado, la densidad de larvas de peces presentó variaciones entre 30 y 1045 ind. $/ \mathrm{m}^{2}$ en los meses de enero y marzo, respectivamente (Fig. 2). 


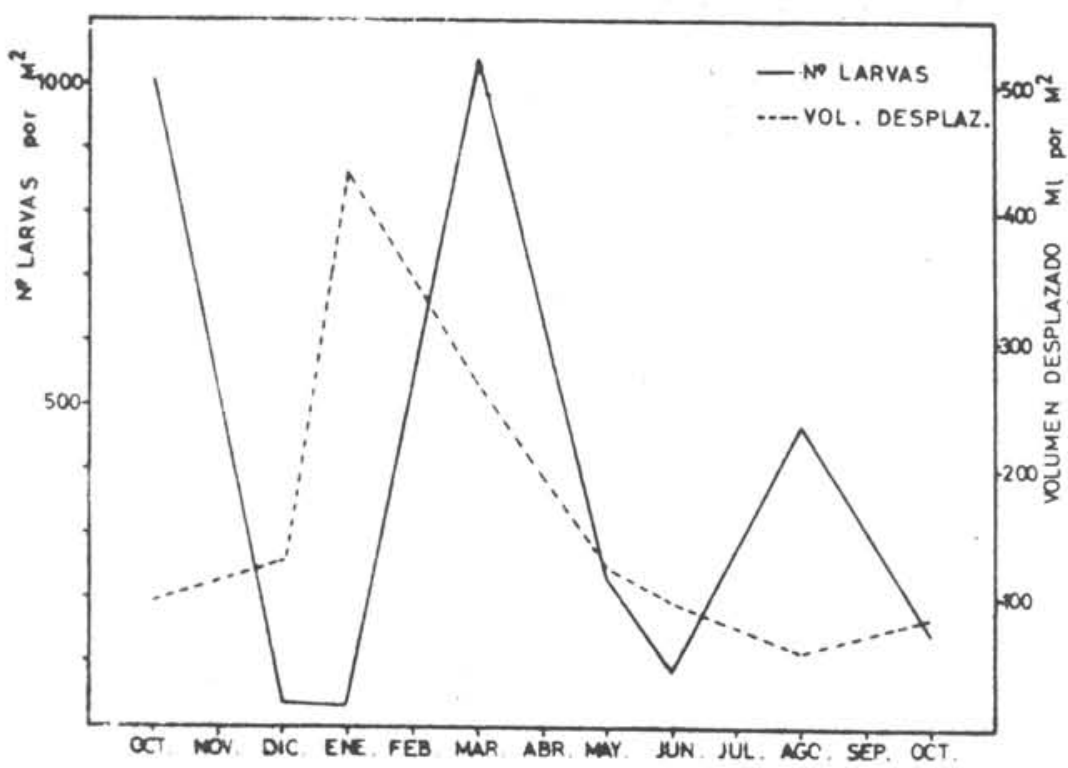

Fig. 2-Grafico de la variación estacional del standing crop zooplanctónico y la densidad de larvas de peces.

Al relacionar la variación anual del SCZ y la densidad de larvas de peces se pudo apreciar que existe una relación inversa; i.e., a un mayor SCZ observado corresponde un menor número de indivíduos de larvas de peces (Fig. 2).

Esta relación ha sido analizada para la costa de California por Murphy (1961) y para el Golfo de Aden por Ali Khan (1974), encontrando la misma relación inversa entre el SCZ y la densidad de las larvas de peces.

La predación del macrozooplancton, v.g., copépodos, hydro. medusas, ctenóforos, ostrácodos y quetognatos y su efecto en la sobrevivencia de las larvas en Bahía de Concepción se puede establecer en forma preliminar. Arcos (1975) establece que existe una mayor densidad de los copépodos calanoídeos en los meses de noviembre, diciembre y enero en Bahía de Concepción, que aunque las muestras no son contemporáneas los ciclos pueden repetirse y observando la Figura 2, podemos darnos cuenta que el número de larvas por $\mathrm{m}^{2}$ desciende precisamente en esos meses

Ahumada (1976) ha caracterizado las aguas de Bahía de Concepción, estableciendo que ellas corresponden a la rama costera del sistema de Humboldt, e.e., Aguas Subantárticas Superficiales. Distingue dos períodos con las correspondientes etapas de transición y que son: Período de circulación estuarina (otoño-invierno) y período de surgencia (primavera-verano). La circulación y transporte de aguas dentro de Bahía de Concepción no está aún bien establecida.

Es, precisamente, en el período de surgencia en el área cuando se observó un $\mathrm{SCZ}$ mayor a $100 \mathrm{ml} / \mathrm{m}^{2}$ en comparación con el periodo de circulación estuarina.

\section{Agradecimientos}

Es nuestro especial interés el reconocer a la Asociación de Industriales Pesqueros de Talcahuano, Chile, quienes, mediante un convenio com la Universidad de Concepción, financian el presente trabajo. Asimismo, a la tripulación del B/C Lund y a todas aquellas personas que colaboraron en las etapas de muestreos.

\section{Referencias bibliograficas}

AHUMADA, E. 1976. Contribución al conocimiento de las condiciones hidrográficas de la Bahía de Concepción y áreas adyacentes. Tesis de grado, Universidad de Concepción. 135 p. Mimeografiada.

ALI KHAN, J. \& HEMPEL G. 1974. Relation of fish larvae and zooplankton biomass in the Gulf to Aden.Mar Biol., 28: 311-316.

ARCOS, D. 1975. Copépodos calanoideos de la Bahía de Concepción, Chile.Conocimiento sistemático y variación estacional. Gayana (Zool.), 32:1-31.

HEMPEL, G. 1965. On the importance of larval survival for the population dynamics of marine food. Rep. Fish. Calif. Coop. ocean. Fish. Invest., 10:13-23.

HJORT, J. 1914. Fluctuations in the great fisheries of northern Europe viewed in the light of biological research. Rapp. Proc. Verb. Cons. perm. int. Explor. Mer, 20:1-28.

MARR, J.C. 1956. The critical period in the early life history of marine fishes. J. Cons. perm. int. Explor. Mer., 21 (2): $160-170$

MAY, R.C. 1974. Larval mortality in marine fishes and the critical period concept. In: The early life history of fish, J.H.S Blaxter (Ed.) Berlin. Springer-Veriag, p. 1-19.

MURPHY, G.I. 1961. Oceanography and variations in the Pacific Sardine population. Rep. Calif. Coop. ocean. Fish. Invest., $8: 55-64$ 